\title{
Development of STEM Imaging in SEM Using Photon Detector
}

Kotaro Hosoya ${ }^{1 *}$, Yukari Dan ${ }^{2}$ and Atsushi Muto ${ }^{1}$

1. Nanotechnology System Division, Hitachi High Technologies America, Inc., Clarksburg, USA.

2. EM Department, Hitachi High Technologies Europe GmbH, Warrington, UK.

* Corresponding author: kotaro.hosoya@hitachi-hta.com

Scanning Transmission Electron Microscopy (STEM) with a scanning electron microscope (SEM) is widely used for semiconductors, nanomaterials and bio specimens for imaging and analysis of internal fine structures.

Until now, there have been two major approaches for STEM imaging by SEM.

The first technique is to utilize a dedicated transmission electron detector. This method has been adopted in ultra-high resolution SEMs for imaging and analysis of angstrom level structures [1].

The second technique is called conversion method [2]. The transmission signal is converted to a secondary electron (SE) and then detected by a conventional SE detector. In this method, signal conversion is achieved by a reflection plate located below the sample. For flexibility, the sample holder / signal conversion plate are integrated into a single unit and due to the fact that SE detectors are universal components for all SEMs, STEM detection capability can be easily obtained with no hardware changes. While this conversion method is very adaptable, low signal yield is a major technical difficulty that is encountered. Low signal yield originates from loss of signal during reflective conversion. Furthermore, the normal (non-reflected) SE signal which contains topographic, compositional, and static information, will generate noise for STEM imaging because these normal SE signals become mixed with reflected SE signal generated by the conversion plate. Furthermore, there is no way to discriminate between these two signals with a conventional SE detector.

In this study, a new STEM observation technique was developed to resolve the technical difficulties presented above. Figure 1 demonstrates a schematic of this novel STEM observation method. By using a conversion type method with a scintillator to generate signal (instead of reflection plate), a photon detector can be used to visualize cathode luminescence (CL) signal. In our study, scintillator showed better signal generation yield than conventional conversion plate and the photon detector eliminated SE signal mixing, which results in improved STEM signal detection as well as better signal-to-noise ratio. Today CL detector technology is commonly used in variable pressure SEMs, such as Ultra Variable detector (UVD) as SE detector in variable pressure mode [3]. The Hitachi UVD functions as a sole photon detector, discriminating SE detection by changing imaging parameters without any hardware modification, so we can maintain the advantage of STEM imaging even if the SEM has CL detector capability.

Figure 2 shows BF-STEM image taken by Dedicated STEM (a) and new STEM scintillator signal conversion (b). The SU5000 schottky SEM is used to obtain both data at same imaging condition with accelerating voltage at $30 \mathrm{kV}$, magnification at $200 \mathrm{kX}$. No significant difference of image quality and sharpness was confirmed between these two analyses, demonstrating the advantage of new technique. 
Figure 2(c) shows same Ag particles observed by SU3500 W-SEM. Even using the STEM scintillator approach with a W filament conventional SEM, high quality STEM images can be captured at 200kX magnification. Since we can keep the probe current low, imaging parameters focused on resolution with high signal yield can be used for this STEM imaging method. STEM imaging applications of various types of materials as well will be also reported.

\section{References:}

[1] Y Orai et al., J. Phys.: Conf. Ser (2014) p.522.

[2] Joseph Goldstein et al., in "Scanning Electron Microscopy and X-ray Microanalysis", (2003) p.203.

[3] M Nishimura et al., Hitachi S.I.NEWS 56 (1) (2013), p.37.

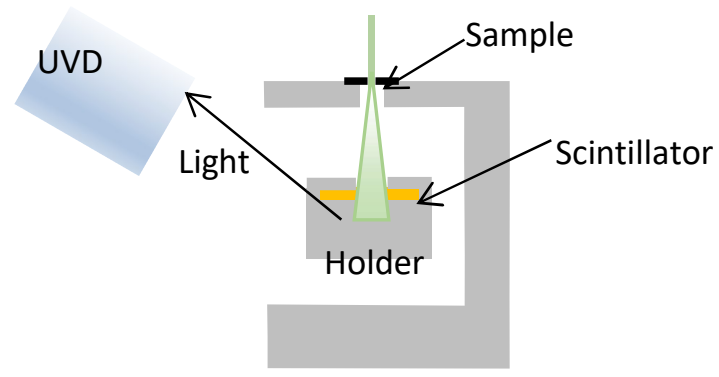

Figure 1. New STEM holder detection system.
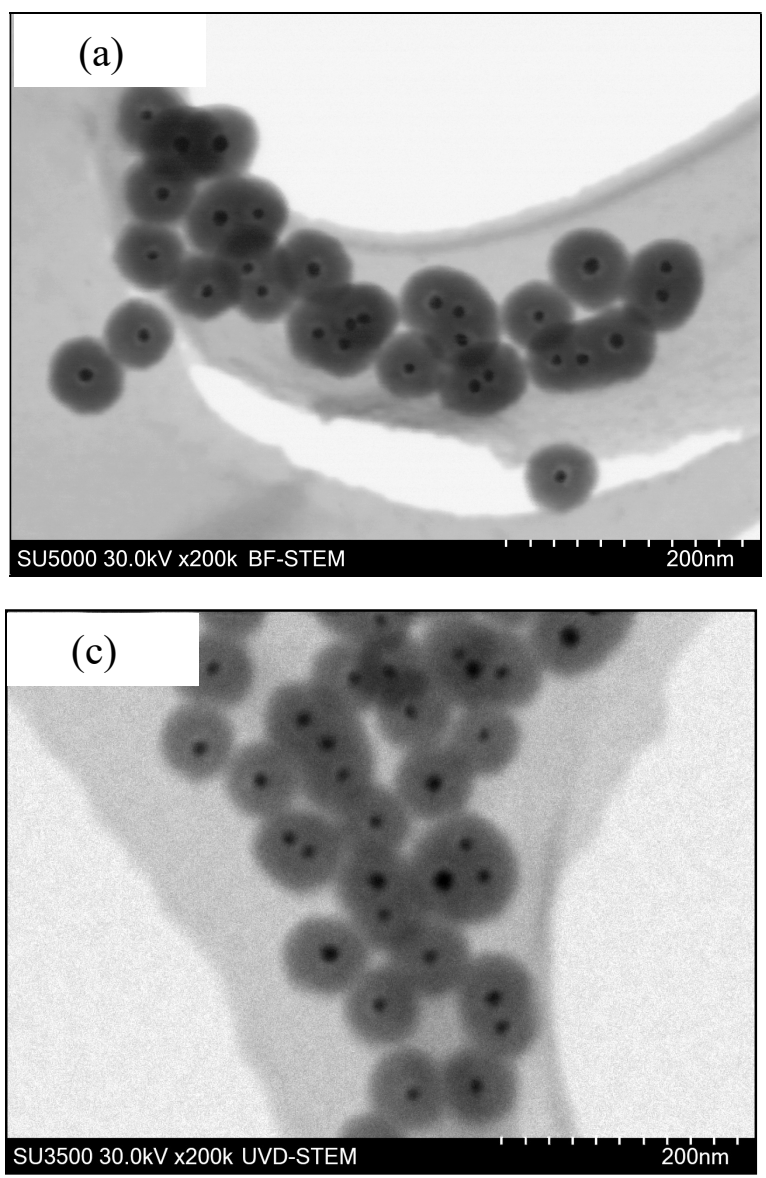

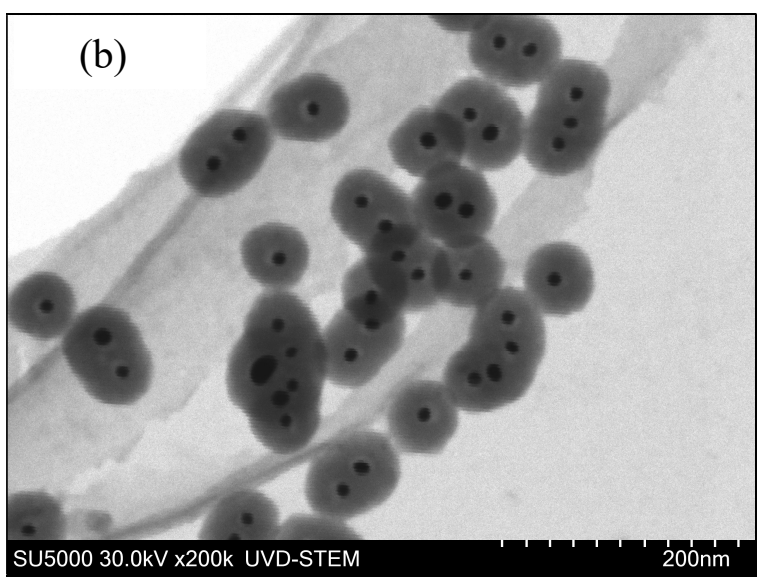

Figure 2. STEM observation of Ag-polymer core-shell nanoparticle

(a) BF-STEM image using dedicated STEM

(b) BF-STEM image using new STEM scintillator signal conversion

(c) BF-STEM image captured by SU3500 W filament Conventional SEM using new STEM scintillator signal conversion 